Shiping Lu* and Xingchen Yu

\title{
Periodic solutions for second order differential equations with indefinite singularities
}

https://doi.org/10.1515/anona-2020-0037

Received February 4, 2019; accepted May 16, 2019.

Abstract: In this paper, the problem of periodic solutions is studied for second order differential equations with indefinite singularities

$$
x^{\prime \prime}(t)+f(x(t)) x^{\prime}(t)+\varphi(t) x^{m}(t)-\frac{\alpha(t)}{x^{\mu}(t)}+\frac{\beta(t)}{x^{y}(t)}=0,
$$

where $f \in C((0,+\infty), \mathbb{R})$ may have a singularity at the origin, the signs of $\varphi$ and $\alpha$ are allowed to change, $m$ is a non-negative constant, $\mu$ and $y$ are positive constants. The approach is based on a continuation theorem of Manásevich and Mawhin with techniques of a priori estimates.

Keywords: Second order differential equation; Continuation theorem; Periodic solution; Indefinite singularity

MSC 2010: 34B16; 34B18; 34C25

\section{Introduction}

In the past years, the problem of existence of periodic solutions to second order differential equations with definite singularities, either attractive type or repulsive type, was extensively studied by many researchers [1]-[14]. In [15], Hakl and Torres investigated the problem of periodic solutions to the equation

$$
\chi^{\prime \prime}(t)=\frac{g(t)}{\chi^{\mu}(t)}-\frac{h(t)}{x^{y}(t)}+r(t),
$$

where $g, h, r$ are $T$-periodic functions with $g, h, r \in L([0, T], \mathbb{R})$. Chu and et. al in [16] studied the problem of twist periodic solutions to (1.1) for the case of $r(t) \equiv 0$. We notice that in [15] and [16], the functions of $g(t)$ and $h(t)$ are required to be $g(t) \geq 0$ and $h(t) \geq 0$ a.e. $t \in[0, T]$. Recently, the periodic problem for second order differential equations with indefinite singularities has attract much attention from researchers (see [12] and [17]-[20]). For example, in [18], the authors considered the existence of positive periodic solutions to the equation like

$$
x^{\prime \prime}(t)=\frac{\alpha(t)}{\chi^{\mu}}
$$

where the sign of weight function $\alpha(t)$ can change on [0,T], and $\mu \geq 1$. The equations like (1.2) with indefinite singularities can be used to model some important problems appearing in many physical contexts (see [21] and the references therein).

*Corresponding Author: Shiping Lu, School of Mathematics and Statistics, Nanjing University of Information Science and Technology, Nanjing, 210044, China, E-mail: lushiping88@sohu.com

Xingchen Yu, School of Mathematics and Statistics, Nanjing University of Information Science and Technology, Nanjing, 210044, China, E-mail: yuxingchen0@yeah.net 
In this paper, we consider the problem of periodic solutions to the equation

$$
x^{\prime \prime}(t)+f(x(t)) x^{\prime}(t)+\varphi(t) x^{m}(t)-\frac{\alpha(t)}{x^{\mu}(t)}+\frac{\beta(t)}{x^{y}(t)}=0,
$$

where $f \in C((0,+\infty), \mathbb{R}), \varphi, \alpha$ and $\beta$ are $T$-periodic and in $L([0, T], \mathbb{R}), m, \mu$ and $y$ are constants with $m \geq 0$ and $\mu \geq y>0$. By using a continuation theorem of Manásevich and Mawhin, some new results on the existence of periodic solutions to (1.3) are obtained. In (1.3), the signs of $\alpha(t), \beta(t)$ and $\varphi(t)$ are all allowed to change. This means that the singularity associated to restoring force $-\frac{\alpha(t)}{\chi^{\mu}}+\frac{\beta(t)}{\chi^{y}}$ at $x=0$ is indefinite type(see $\left.[18,19]\right)$. The periodic problem for equation (1.3) has been investigated in recent paper [20]. However, in [20], $\beta(t) \equiv 0$, and the functions of $\alpha(t)$ and $\varphi(t)$ are required to be either $\alpha(t) \geq 0$ a.e. $t \in[0, T], \bar{\alpha}>0$ and $\bar{\varphi}>0$, or $\varphi(t) \geq 0$ a.e. $t \in[0, T], \bar{\alpha}>0$ and $\bar{\varphi}>0$. The significance of present paper relies in the following aspects. Firstly, the coefficient function $f(x)$ associated to friction term $f(x) x^{\prime}$ may have a singularity at $x=0$. Secondly, compared with the case where the signs of functions $\varphi(t), \alpha(t)$ and $\beta(t)$ are in definite, the work of obtaining the estimates of periodic solutions to (1.3) is more difficult. In order to overcome this difficulty, we propose a function $F(x)=\int_{1}^{x} f(s) d s$. By analyzing some properties of $F(x)$ at $x=0$ and $x=+\infty$, we investigate the mechanism under which how the singularity associated to $f(x)$ at $x=0$ influences the priori estimates of periodic solutions. Moreover, the constant $\mu$ in (1.3) is allowed to be in $(0,1)$. For this case, even if $\alpha(t)$ and $\beta(t)$ are constant functions, the singular restoring force $-\frac{\alpha(t)}{x^{\mu}}+\frac{\beta(t)}{x^{y}}$ has a weak singularity at $x=0$. Finally, by using a theorem in present paper, a new result on the existence of periodic solutions is obtained for RayleighPlesset equation

$$
\rho\left(R R^{\prime \prime}+\frac{3}{2}\left(R^{\prime}\right)^{2}\right)=\left[P_{v}-P_{\infty}(t)\right]+P_{g_{0}}\left(\frac{R_{0}}{R}\right)^{3 k}-\frac{2 S}{R}-\frac{4 v R^{\prime}}{R}
$$

in the case of $k \in\left(\frac{1}{3},+\infty\right)$. Equation (1.4) is used in physics of fluids to model the oscillations of the radius $R(t)$ of a spherical bubble immersed in a fluid under the influence of a periodic acoustic field $P_{\infty}$ (see [21]-[25]), and $P_{\infty} \in L([0, T], \mathbb{R})$. The physical meaning of the rest of the parameters in (1.4) can also be seen in Section 3 of [5]. By using the methods of upper and lower solutions, the authors in [5] obtained the following result:

Theorem 1.1. Suppose that $P_{v}>\overline{P_{\infty}}$ and

$$
\frac{5}{2 \rho}\left(P_{v}-P_{\infty}(t)\right) \leq\left(\frac{6 k-2}{5}\right)\left[\frac{\left(\frac{2}{5}\right)^{\frac{2}{5}}(5 S)^{\frac{6 k}{5}}}{\left(\frac{6 k}{5}\right)^{\frac{6 k}{5}}\left(\frac{5 P_{g_{0}} R_{0}^{3 k}}{2 \rho}\right)^{\frac{6 k-2}{5}}}\right]^{\frac{5}{6 k-2}}, \text { for } t \in[0, T] .
$$

Then there exists at least one positive T-periodic solution to the Rayleigh-Plesset equation (1.4) in the case of $k \in\left(\frac{1}{3},+\infty\right)$.

\section{Preliminaries}

Throughout this paper, let $C_{T}=\{x \in C(\mathbb{R}, \mathbb{R}): x(t+T)=x(t)$ for all $t \in \mathbb{R}\}$ with the norm defined by $|x|_{\infty}=\max _{t \in[0, T]}|x(t)|$, and $C_{T}^{1}=\left\{x \in C^{1}(\mathbb{R}, \mathbb{R}): x(t+T)=x(t)\right.$ for all $\left.t \in \mathbb{R}\right\}$ with the norm defined by $\|x\|_{C_{T}^{1}}=\max \left\{|x|_{\infty},\left|x^{\prime}\right|_{\infty}\right\}$. For any $T$-periodic function $y(t)$ with $y \in L([0, T], \mathbb{R}), y_{+}(t)$ and $y_{-}(t)$ is denoted by $\max \{y(t), 0\}$ and $-\min \{y(t), 0\}$, respectively, and $\bar{y}=\frac{1}{T} \int_{0}^{T} y(s) d s$. Clearly, $y(t)=y_{+}(t)-y_{-}(t)$ for all $t \in \mathbb{R}$, and $\bar{y}=\overline{y_{+}}-\overline{y_{-}}$.

Lemma 2.1 ([20, 26]). Assume that there exist positive constants $M_{0}, M_{1}$ and $M_{2}$ with $0<M_{0}<M_{1}$, such that the following conditions hold.

1.For any $\lambda \in(0,1]$, each possible positive $T$-periodic solution $x$ to the equation

$$
u^{\prime \prime}(t)+\lambda f(u(t)) u^{\prime}(t)+\lambda \varphi(t) u^{m}(t)-\frac{\lambda \alpha(t)}{\chi^{\mu}(t)}+\frac{\lambda \beta(t)}{\chi^{y}(t)}=0
$$


satisfies the inequalities $M_{0}<x(t)<M_{1}$ and $\left|\chi^{\prime}(t)\right|<M_{2}$, for all $t \in[0, T]$.

2.Each possible solution $c$ to the equation

$$
\frac{\bar{\alpha}}{c^{\mu}}-\frac{\bar{\beta}}{c^{y}}-\bar{\varphi} c^{m}=0
$$

satisfies the inequality $M_{0}<c<M_{1}$.

3.The inequality

$$
\left(\frac{\bar{\alpha}}{M_{0}^{\mu}}-\frac{\bar{\beta}}{M_{0}^{y}}-\bar{\varphi} M_{0}^{m}\right)\left(\frac{\bar{\alpha}}{M_{1}^{\mu}}-\frac{\bar{\beta}}{M_{1}^{y}}-\bar{\varphi} M_{1}^{m}\right)<0
$$

holds.

Then, equation (1.3) has at least one T-periodic solution u such that $M_{0}<u(t)<M_{1}$ for all $t \in[0, T]$.

Remark 2.1 If $\bar{\varphi}>0$ and $\bar{\alpha}>0$, then we have from the condition of $m \geq 0$ and $\mu>y>0$ that there are two constants $D_{1}$ and $D_{2}$ with $0<D_{1}<D_{2}$ such that

$$
\frac{\bar{\alpha}}{\chi^{\mu}}-\frac{\bar{\beta}}{\chi^{y}}-\bar{\varphi} x^{m}>0 \text {, for all } x \in\left(0, D_{1}\right)
$$

and

$$
\frac{\bar{\alpha}}{\chi^{\mu}}-\frac{\bar{\beta}}{\chi^{y}}-\bar{\varphi} x^{m}<0 \text {, for all } x \in\left(D_{2}, \infty\right) .
$$

Lemma 2.2 ([5]). Let $u \in[0, \omega] \rightarrow \mathbb{R}$ be an arbitrary absolutely continuous function with $u(0)=u(\omega)$.Then the inequality

$$
\left(\max _{t \in[0, \omega]} u(t)-\min _{t \in[0, \omega]} u(t)\right)^{2} \leq \frac{\omega}{4} \int_{4}^{\omega}\left|u^{\prime}(s)\right|^{2} d s
$$

holds.

Now, we embed equation (1.3) into the following equations family with a parameter $\lambda \in(0,1]$

$$
x^{\prime \prime}+\lambda f(x) x^{\prime}+\lambda \varphi(t) x^{m}-\frac{\lambda \alpha(t)}{x^{\mu}}=0, \lambda \in(0,1] .
$$

Let

$$
\begin{gathered}
D=\left\{x \in C_{T}^{1}: x^{\prime \prime}+\lambda f(x) x^{\prime}+\lambda \varphi(t) x^{m}-\frac{\lambda \alpha(t)}{x^{\mu}}+\frac{\lambda \beta(t)}{u^{y}(t)}=0,\right. \\
\lambda \in(0,1] ; x(t)>0, \forall t \in[0, T]\}, \\
F(x)=\int_{1}^{x} f(s) d s
\end{gathered}
$$

and

$$
G(x)=\int_{1}^{x} s^{\mu} f(s) d s .
$$

Lemma 2.3. Assume $\bar{\alpha}>0$ and $\bar{\varphi}>0$, then for each $u \in D$, there are constants $\tau_{1}, \tau_{2} \in[0, T]$ such that

$$
u\left(\tau_{1}\right) \leq \max \left\{1,\left(\frac{\overline{\alpha_{+}}+\overline{\beta_{-}}}{\bar{\varphi}}\right)^{\frac{1}{m+y}}\right\}:=A_{0}
$$

and

$$
u\left(\tau_{2}\right) \geq A_{1},
$$

where

$$
A_{1}= \begin{cases}\min \left\{\left(\frac{\bar{\alpha}}{2 \overline{\varphi_{+}}}\right)^{\frac{1}{\mu+m}},\left(\frac{\bar{\alpha}}{2 \overline{\beta_{+}}}\right)^{\frac{1}{\mu-y}}\right\}, & \overline{\beta_{+}}>0 \\ \left(\frac{\bar{\alpha}}{\bar{\varphi}_{+}}\right)^{\frac{1}{\mu+m}}, & \overline{\beta_{+}}=0,\end{cases}
$$

$m, \mu$ and $y$ are determined in equation (1.3) 
Proof. Let $u \in D$, then

$$
u^{\prime \prime}(t)+\lambda f(u(t)) u^{\prime}(t)+\lambda \varphi(t) u^{m}(t)-\frac{\lambda \alpha(t)}{u^{\mu}(t)}+\frac{\lambda \beta(t)}{u^{y}(t)}=0,
$$

which together with the fact of $u(t)>0$ for all $t \in[0, T]$ gives

$$
\frac{u^{\prime \prime}}{u^{m}}+\frac{\lambda f(u) u^{\prime}}{u^{m}}+\lambda \varphi(t)-\frac{\lambda \alpha(t)}{u^{m+\mu}}+\frac{\lambda \beta(t)}{u^{y+m}(t)}=0 .
$$

Integrating the above equality over the interval $[0, T]$, we obtain

$$
\int_{0}^{T} \frac{u^{\prime \prime}}{u^{m}} d t+\lambda \int_{0}^{T} \varphi(t) d t=\lambda \int_{0}^{T} \frac{\alpha(t)}{u^{m+\mu}} d t-\lambda \int_{0}^{T} \frac{\beta(t)}{u^{y+m}(t)} d t
$$

i.e.,

$$
\int_{0}^{T} \frac{u^{\prime \prime}}{u^{m}} d t+\lambda T \bar{\varphi}=\lambda \int_{0}^{T} \frac{\alpha(t)}{u^{m+\mu}} d t-\lambda \int_{0}^{T} \frac{\beta(t)}{u^{y+m}(t)} d t
$$

Since

$$
\int_{0}^{T} \frac{u^{\prime \prime}}{u^{m}} d t \geq 0
$$

it follows from (2.9) that

$$
T \bar{\varphi} \leq \int_{0}^{T} \frac{\alpha(t)}{u^{m+\mu}} d t-\int_{0}^{T} \frac{\beta(t)}{u^{y+m}(t)} d t \leq \int_{0}^{T} \frac{\alpha_{+}(t)}{u^{m+\mu}(t)} d t+\int_{0}^{T} \frac{\beta_{-}(t)}{u^{y+m}(t)} d t .
$$

From (2.11), we can conclude that there is a point $\xi \in[0, T]$ such that

$$
u(\xi) \leq \max \left\{1,\left(\frac{\overline{\alpha_{+}}+\overline{\beta_{-}}}{\bar{\varphi}}\right)^{\frac{1}{m^{++}}}\right\} .
$$

If (2.12) does not hold, then

$$
u(t)>\max \left\{1,\left(\frac{\overline{\alpha_{+}}+\overline{\beta_{-}}}{\bar{\varphi}}\right)^{\frac{1}{m+y}}\right\} \text { for all } t \in[0, T]
$$

which implies that

$$
u(t)>1 \text { for all } t \in[0, T]
$$

and

$$
u(t)>\left(\frac{\overline{\alpha_{+}}+\overline{\beta_{-}}}{\bar{\varphi}}\right)^{\frac{1}{m+y}} \text { for all } t \in[0, T]
$$

From (2.11), (2.13) and $\mu>y$, and by using mean value theorem of integrals, we have that there exists a point $\eta \in[0, T]$ such that

$$
T \bar{\varphi} \leq \frac{T \overline{\alpha_{+}}+T \overline{\beta_{-}}}{u^{m+y}(\eta)}
$$

i.e.,

$$
u(\eta) \leq\left(\frac{\overline{\alpha_{+}}+\overline{\beta_{-}}}{\bar{\varphi}}\right)^{\frac{1}{m+y}}
$$

which contradicts to (2.14). This contradiction verifies (2.12), and so (2.5) holds. Meanwhile, we can assert that (2.6) is true. In fact, multiplying two sides of (2.8) with $u^{\mu}(t)$ and integrating it over the interval $[0, T]$, we obtain that

$$
-\int_{0}^{T} u^{\mu}(t) u^{\prime \prime}(t) d t-\lambda \int_{0}^{T} \varphi(s) u^{\mu+m} d s-\lambda \int_{0}^{T} \beta(t) u^{\mu-y}(t) d t+\lambda T \bar{\alpha}=0,
$$


which together with

$$
-\int_{0}^{T} u^{\mu}(t) u^{\prime \prime}(t) d t=\int_{0}^{T} u^{\mu-1}(s)\left|u^{\prime}(s)\right|^{2} d t \geq 0
$$

yields

$$
T \bar{\alpha} \leq \int_{0}^{T} \varphi(s) u^{\mu+m} d s+\int_{0}^{T} \beta(t) u^{\mu-y}(t) d t \leq \int_{0}^{T} \varphi_{+}(s) u^{\mu+m}(s) d s+\int_{0}^{T} \beta_{+}(t) u^{\mu-y}(t) d t .
$$

Thus, there is a point $\eta \in[0, T]$ such that

$$
u(\eta) \geq A_{1},
$$

where $A_{1}$ is defined by (2.7). This implies that (2.6) holds.

Lemma 2.4. Assume $\bar{\alpha}>0$ and $\bar{\varphi}>0$, and suppose that the following assumptions

$$
C_{0}:=\sup _{x \in\left[A_{1},+\infty\right)}\left[F(x)+T \overline{\varphi_{-}} x^{m}\right]<+\infty,
$$

and

$$
\lim _{s \rightarrow 0^{+}}\left(F(s)-\frac{T \overline{\alpha_{-}}}{s^{\mu}}-\frac{T \overline{\beta_{+}}}{s^{y}}\right)>C_{0}
$$

hold, where $F(x)$ is defined by (2.3).

Then there exists a constant $y_{0}>0$, such that

$$
\min _{t \in[0, T]} u(t) \geq y_{0}, \text { uniformly for } u \in D .
$$

Proof. For each $u \in D$, we have

$$
u^{\prime \prime}(t)+\lambda f(u(t)) u^{\prime}(t)+\lambda \varphi(t) u^{m}(t)-\frac{\lambda \alpha(t)}{u^{\mu}(t)}+\frac{\lambda \beta(t)}{u^{y}(t)}=0, \quad \lambda \in(0,1] .
$$

Since $u(t)$ is a $T$-periodic function, there are two points $t_{1}, t_{2} \in \mathbb{R}$ such that $0<t_{2}-t_{1} \leq T$ with $u\left(t_{1}\right)=$ $\max _{t \in[0, T]} u(t)$ and $u\left(t_{2}\right)=\min _{t \in[0, T]} u(t)$. Assumptions of $\bar{\alpha}>0$ and $\bar{\varphi}>0$ implies that (2.6) in Lemma 2.3 holds. This gives

$$
A_{1} \leq u\left(t_{1}\right)<+\infty,
$$

to which by applying assumption (2.16) yields

$$
F\left(u\left(t_{1}\right)\right)+T \overline{\varphi_{-}} u^{m}\left(t_{1}\right) \leq \sup _{A_{1} \leq s<+\infty}\left[F(s)+T \overline{\varphi_{-}} s^{m}\right]:=C_{0}<+\infty .
$$

By integrating (2.18) over the interval $\left[t_{1}, t_{2}\right]$, we get

$$
\begin{aligned}
& \int_{t_{1}}^{t_{2}} f(u(t)) u^{\prime}(t) d t \\
& =\int_{t_{1}}^{t_{2}} \frac{\alpha(t)}{u^{\mu}(t)} d t-\int_{t_{1}}^{t_{2}} \frac{\beta(t)}{u^{y}(t)} d t-\int_{t_{1}}^{t_{2}} \varphi(t) u^{m}(t) d t .
\end{aligned}
$$

It follows from (2.19), (2.20) and the condition $\bar{\varphi}>0$ that

$$
\begin{aligned}
F\left(u\left(t_{2}\right)\right) & =F\left(u\left(t_{1}\right)\right)-\int_{t_{1}}^{t_{2}} \varphi(t) u^{m}(t) d t+\int_{t_{1}}^{t_{2}} \frac{\alpha(t)}{u^{\mu}(t)} d t-\int_{t_{1}}^{t_{2}} \frac{\beta(t)}{u^{y}(t)} d t \\
& \leq F\left(u\left(t_{1}\right)\right)+\int_{0}^{T} \varphi_{-}(t) u^{m}(t) d t+\int_{0}^{T} \frac{\alpha_{+}(t)}{u^{\mu}(t)} d t+\int_{0}^{T} \frac{\beta_{-}(t)}{u^{y}(t)} d t .
\end{aligned}
$$


On the other hand, by integrating $(2.18)$ on $[0, T]$, we get

$$
\int_{0}^{T} \frac{\alpha(t)}{u^{\mu}(t)} d t=\int_{0}^{T} \frac{\beta(t)}{u^{y}(t)} d t+\int_{0}^{T} \varphi(t) u^{m}(t) d t,
$$

i.e.,

$$
\begin{gathered}
\int_{0}^{T} \frac{\alpha_{+}(t)}{u^{\mu}(t)} d t+\int_{0}^{T} \frac{\beta_{-}(t)}{u^{y}(t)} d t+\int_{0}^{T} \varphi_{-}(t) u^{m}(t) d t \\
=\int_{0}^{T} \frac{\alpha_{-}(t)}{u^{\mu}(t)} d t+\int_{0}^{T} \frac{\beta_{+}(t)}{u^{y}(t)} d t+\int_{0}^{T} \varphi_{+}(t) u^{m}(t) d t .
\end{gathered}
$$

Substituting it into (2.21), we have from (2.19) that

$$
\begin{aligned}
& \quad F\left(u\left(t_{2}\right)\right) \\
& \leq F\left(u\left(t_{1}\right)\right)+\int_{0}^{T} \frac{\alpha_{-}(t)}{u^{\mu}(t)} d t+\int_{0}^{T} \frac{\beta_{+}(t)}{u^{y}(t)} d t+\int_{0}^{T} \varphi_{+}(t) u^{m}(t) d t \\
& \leq F\left(u\left(t_{1}\right)\right)+T \overline{\varphi_{+}} u^{m}\left(t_{1}\right)+\frac{T \overline{\alpha_{-}}}{u^{\mu}\left(t_{2}\right)}+\frac{T \overline{\beta_{+}}}{u^{y}\left(t_{2}\right)} \\
& \leq C_{0}+\frac{T \overline{\alpha_{-}}}{u^{\mu}\left(t_{2}\right)}+\frac{T \overline{\beta_{+}}}{u^{y}\left(t_{2}\right)},
\end{aligned}
$$

and so

$$
F\left(u\left(t_{2}\right)\right)-\frac{T \overline{\alpha_{-}}}{u^{\mu}\left(t_{2}\right)}-\frac{T \overline{\beta_{+}}}{u^{y}\left(t_{2}\right)} \leq C_{0} .
$$

Assumption (2.17) ensures that there exists a constant $y_{0}>0$ such that

$$
F(s)-\frac{T \overline{\alpha_{-}}}{s^{\mu}}-\frac{T \overline{\beta_{+}}}{s^{y}}>C_{0} \text {, for all } s \in\left(0, y_{0}\right) .
$$

Combining (2.23) with (2.22), we can get

$$
\min _{t \in[0, T]} u(t)=u\left(t_{2}\right) \geq y_{0}
$$

Lemma 2.5. Assume $\bar{\alpha}>0$ and $\bar{\varphi}>0$. Suppose further that the following assumptions

$$
B_{0}:=\inf _{x \in\left[A_{1},+\infty\right)}\left[F(x)-T \overline{\varphi_{+}} x^{m}\right]>-\infty
$$

and

$$
\lim _{s \rightarrow 0^{+}}\left(F(s)+\frac{T \overline{\alpha_{-}}}{s^{\mu}}+\frac{T \overline{\beta_{+}}}{s^{y}}\right)<B_{0}
$$

hold. Then there exists a constant $y_{1}>0$, such that

$$
\min _{t \in[0, T]} u(t) \geq y_{1} \text {, uniformly for } u \in D
$$

Proof. Suppose that $u \in D$, then $u$ satisfies (2.18). Let $t_{1}$ and $t_{2}$ be defined as same as the ones in the proof of Lemma 2.4. From (2.6) in Lemma 2.3, we see that

$$
A_{1} \leq u\left(t_{1}\right)<+\infty
$$


which together with assumption (2.25), yields that

$$
F\left(u\left(t_{1}\right)\right)-T \overline{\varphi_{+}} u^{m}\left(t_{1}\right) \geq \inf _{A_{1} \leq s<+\infty}\left[F(s)-T \overline{\varphi_{+}} s^{m}\right]:=B_{0} .
$$

By integrating (2.18) over the interval $\left[t_{1}, t_{2}\right]$, we get

$$
\begin{aligned}
& F\left(u\left(t_{2}\right)\right) \\
& =F\left(u\left(t_{1}\right)\right)+\int_{t_{1}}^{t_{2}} \frac{\alpha(t)}{u^{\mu}(t)} d t-\int_{t_{1}}^{t_{2}} \frac{\beta(t)}{u^{y}(t)} d t-\int_{t_{1}}^{t_{2}} \varphi(t) u^{m}(t) d t \\
& \geq F\left(u\left(t_{1}\right)\right)-\int_{0}^{T} \frac{\alpha_{-}(t)}{u^{\mu}(t)} d t-\int_{0}^{T} \frac{\beta_{+}(t)}{u^{y}(t)} d t-\int_{0}^{T} \varphi_{+}(t) u^{m}(t) d t \\
& \geq F\left(u\left(t_{1}\right)\right)-\frac{T \overline{\alpha_{-}}}{u^{\mu}\left(t_{2}\right)}-\frac{T \overline{\beta_{+}}}{u^{y}\left(t_{2}\right)}-T \overline{\varphi_{+}} u^{m}\left(t_{1}\right),
\end{aligned}
$$

i.e.,

$$
F\left(u\left(t_{2}\right)\right)+\frac{T \alpha_{-}}{u^{\mu}\left(t_{2}\right)}-\frac{T \overline{\beta_{+}}}{u^{y}\left(t_{2}\right)} \geq F\left(u\left(t_{1}\right)\right)-T \overline{\varphi_{+}} u^{m}\left(t_{1}\right)
$$

From (2.27), we obtain

$$
F\left(u\left(t_{2}\right)\right)+\frac{T \alpha_{-}}{u^{\mu}\left(t_{2}\right)}+\frac{T \overline{\beta_{+}}}{u^{y}\left(t_{2}\right)} \geq B_{0} .
$$

Assumption (2.26) implies that there exists a constant $y_{1}>0$ such that

$$
F(s)+\frac{T \overline{\alpha_{-}}}{s^{\mu}}+\frac{T \overline{\beta_{+}}}{s^{y}}<B_{0} \text {, for all } s \in\left(0, y_{1}\right),
$$

which together with (2.28) gives

$$
\min _{t \in[0, T]} u(t)=u\left(t_{2}\right) \geq y_{1}
$$

\section{Main Results}

Theorem 3.1. Assume $\bar{\varphi}>0$ and $\bar{\alpha}>0$. Suppose further

$$
\lim _{s \rightarrow 0^{+}}\left(F(s)-\frac{T \overline{\alpha_{+}}}{s^{\mu}}-\frac{T \overline{\beta_{-}}}{s^{y}}\right)=+\infty
$$

and

$$
\lim _{x \rightarrow+\infty}\left(F(x)+T \overline{\varphi_{+}} x^{m}\right)=-\infty .
$$

Then equation(1.3) has at least one positive T-periodic solution.

Proof. Firstly, we will prove that there exist two constants $y_{2}>0$ and $y_{3}>0$ with $y_{3}>y_{2}$, such that

$$
\min _{t \in[0, T]} u(t) \geq y_{2} \text {, uniformly for } u \in D
$$

and

$$
\max _{t \in[0, T]} u(t) \leq y_{3}, \text { uniformly for } u \in D .
$$

For each $u \in D$, $u$ satisfies (2.18). Let $t_{1}$ and $t_{2}$ be defined as same as the ones in the proof of Lemma 2.4, that is $0<t_{2}-t_{1} \leq T, u\left(t_{1}\right)=\max _{t \in[0, T]} u(t)$ and $u\left(t_{2}\right)=\min _{t \in[0, T]} u(t)$. Take $t_{3}=t_{1}+T$, then $u\left(t_{3}\right)=\max _{t \in[0, T]} u(t)$ 
and $0 \leq t_{3}-t_{2}<T$. It is easy to see that (2.16) can be deduced from (3.2), and (3.1) verifies (2.17). By using Lemma 2.4, as well as (2.5) in Lemma 2.3, we know that

$$
A_{0} \geq \min _{t \in[0, T]} u(t)=u\left(t_{2}\right) \geq y_{0} .
$$

Integrating (2.18) over the interval $\left[t_{2}, t_{3}\right]$, we have

$$
\begin{aligned}
& F\left(u\left(t_{3}\right)\right) \\
&= F\left(u\left(t_{2}\right)\right)+\int_{t_{2}}^{t_{3}} \frac{\alpha(t)}{u^{\mu}(t)} d t-\int_{t_{2}}^{t_{3}} \frac{\beta(t)}{u^{y}(t)} d t-\int_{t_{2}}^{t_{3}} \varphi(t) u^{m}(t) d t \\
& \geq F\left(u\left(t_{2}\right)\right)-\int_{0}^{T} \frac{\alpha_{-}(t)}{u^{\mu}(t)} d t-\int_{0}^{T} \frac{\beta_{+}(t)}{u^{y}(t)} d t-\int_{0}^{T} \varphi_{+}(t) u^{m}(t) d t \\
& \geq F\left(u\left(t_{2}\right)\right)-\frac{T \overline{\alpha_{-}}}{u^{\mu}\left(t_{2}\right)}-\frac{T \overline{\beta_{+}}}{u^{y}\left(t_{2}\right)}-T \overline{\varphi_{+}} u^{m}\left(t_{3}\right),
\end{aligned}
$$

i.e.,

$$
F\left(u\left(t_{3}\right)\right)+T \overline{\varphi_{+}} u^{m}\left(t_{3}\right) \geq F\left(u\left(t_{2}\right)\right)-\frac{T \overline{\alpha_{-}}}{u^{\mu}\left(t_{2}\right)}-\frac{T \overline{\beta_{+}}}{u^{y}\left(t_{2}\right)},
$$

which together with (3.5) gives

$$
F\left(u\left(t_{3}\right)\right)+T \overline{\varphi_{+}} u^{m}\left(t_{3}\right) \geq \inf _{x \in\left[y_{0}, A_{0}\right]}\left[F(x)-\frac{T \overline{\alpha_{-}}}{x^{\mu}}-\frac{T \overline{\beta_{+}}}{x^{y}}\right]:=\rho_{0}>-\infty .
$$

It follows from (3.2) that there is a constant $y_{3}>0$ such that

$$
\max _{t \in[0, T]} u(t)=u\left(t_{3}\right) \leq y_{3} .
$$

From (3.5) and (3.6), we see that the conclusions of (3.3) and (3.4) hold. Next, we will show that there exists a positive constant $\rho_{1}$ such that

$$
\max _{t \in[0, T]}\left|u^{\prime}(t)\right| \leq \rho \text {, uniformly for } u \in D \text {. }
$$

In fact, if $u \in D$, then

$$
u^{\prime \prime}(t)+\lambda f(u(t)) u^{\prime}(t)+\lambda \varphi(t) u^{m}(t)-\frac{\lambda \alpha(t)}{u^{\mu}(t)}+\frac{\lambda \beta(t)}{u^{y}(t)}=0, \lambda \in(0,1]
$$

Let $u$ attain its maximum over $[0, T]$ at $t_{1} \in[0, T]$, then $u^{\prime}\left(t_{1}\right)=0$ and we deduce from (3.8) that

$$
u^{\prime}(t)=\lambda \int_{t_{1}}^{t}\left[-f(u(s)) u^{\prime}(s)-\varphi(s) u^{m}(s)+\frac{\alpha(s)}{u^{\mu}(s)}-\frac{\beta(s)}{u^{y}(s)}\right] d s
$$

for all $t \in\left[t_{1}, t_{1}+T\right]$. Thus,

$$
\begin{aligned}
&\left|u^{\prime}(t)\right| \leq \lambda\left|F(u(t))-F\left(u\left(t_{1}\right)\right)\right|+\lambda \int_{t_{1}}^{t_{1}+T}\left|\frac{\alpha(s)}{u^{\mu}(s)}\right| d s+\lambda \int_{t_{1}}^{t_{1}+T}\left|\frac{\beta(s)}{u^{y}(s)}\right| d s \\
&+\int_{t_{1}}^{t_{1}+T}|\varphi(s)| u^{m}(s) d s \\
& \leq 2 \max _{y_{2} \leq u \leq y_{3}}|F(u)|+\int_{0}^{T}\left|\frac{\alpha(t)}{u^{\mu}(t)}\right| d t+\int_{0}^{T}\left|\frac{\beta(t)}{u^{y}(t)}\right| d t+\int_{0}^{T}|\varphi(s)| u^{m}(s) d s .
\end{aligned}
$$


It follows from (3.4) and (3.3) that

$$
\begin{aligned}
\left|u^{\prime}(t)\right| & \leq 2 \max _{y_{0} \leq u \leq y_{3}}|F(u)|+\frac{T \overline{|\alpha|}}{y_{1}^{\mu}}+\frac{T \overline{|\beta|}}{y_{1}^{y}}+T y_{3}^{m} \overline{|\varphi|} \\
& :=\rho, \text { for all } t \in[0, T],
\end{aligned}
$$

and so

$$
\max _{t \in[0, T]}\left|u^{\prime}(t)\right| \leq \rho, \text { uniformly for } u \in D,
$$

which implies that (3.7) holds. Let $m_{0}=\min \left\{D_{1}, y_{2}\right\}$ and $m_{1}=\max \left\{y_{3}, D_{2}\right\}$ be two constants, where $D_{1}$ and $D_{2}$ are constants determined in Remark 2.1, then from (3.4), (3.3) and (3.7), we see that each possible positive $T$-periodic solution $u$ to equation(2.1) satisfies

$$
m_{0}<u(t)<m_{1},\left|u^{\prime}(t)\right|<\rho_{1} \text { for all } t \in[0, T]
$$

This implies that condition 1 of Lemma 2.1 is satisfied. Also, we can deduce from Remark 2.1 that

$$
\frac{\bar{\alpha}}{\chi^{\mu}}-\frac{\bar{\beta}}{x^{y}}-\bar{\varphi} x^{m}>0, \text { for } x \in\left(0, m_{0}\right]
$$

and

$$
\frac{\bar{\alpha}}{x^{\mu}}-\frac{\bar{\beta}}{x^{y}}-\bar{\varphi} x^{m}<0, \text { for } x \in\left[m_{1},+\infty\right),
$$

which gives that condition 2 of Lemma 2.1 holds. Furthermore, we have

$$
\left(\frac{\bar{\alpha}}{m_{0}^{\mu}}-\frac{\bar{\beta}}{m_{0}^{y}}-\bar{\varphi} m_{0}^{m}\right)\left(\frac{\bar{\alpha}}{m_{1}^{\mu}}-\frac{\bar{\beta}}{m_{1}^{y}}-\bar{\varphi} m_{1}^{m}\right)<0 .
$$

So condition 3 of Lemma 2.1 holds, too. By using Lemma 2.1, we see that equation(1.3) has at least one positive $T$-periodic solution.

Theorem 3.2. Assume $\bar{\varphi}>0$ and $\bar{\alpha}>0$. Suppose further

$$
\lim _{s \rightarrow 0^{+}}\left(F(s)+\frac{T \overline{\alpha_{+}}}{s^{\mu}}+\frac{T \overline{\beta_{-}}}{s^{y}}\right)=-\infty
$$

and

$$
\lim _{x \rightarrow+\infty}\left(F(x)-T \overline{\varphi_{+}} x^{m}\right)=+\infty .
$$

Then equation(1.3) has at leat one positive T-periodic solution.

Proof. From the proof of Theorem 3.1, we see that it suffices for us to verify (3.4) and (3.3). In order to do it, let $u \in D, u$ satisfies (2.18). Let $t_{2}$ and $t_{3}$ be defined as same as the ones in the proof of Theorem 3.1, that is $0 \leq t_{3}-t_{2}<T, u\left(t_{3}\right)=\max _{t \in[0, T]} u(t)$ and $u\left(t_{2}\right)=\min _{t \in[0, T]} u(t)$. It is easy to see that (2.25) can be deduced from (3.10), and (3.9) verifies (2.26). By using Lemma 2.5 and (2.5) in Lemma 2.4, we know that

$$
A_{0} \geq \min _{t \in[0, T]} u(t)=u\left(t_{2}\right) \geq y_{1} .
$$

Integrating (2.18) over the interval $\left[t_{2}, t_{3}\right]$, we have

$$
\begin{aligned}
& F\left(u\left(t_{3}\right)\right) \\
= & F\left(u\left(t_{2}\right)\right)+\int_{t_{2}}^{t_{3}} \frac{\alpha(t)}{u^{\mu}(t)} d t-\int_{t_{2}}^{t_{3}} \frac{\beta(t)}{u^{y}(t)} d t-\int_{t_{2}}^{t_{3}} \varphi(t) u^{m}(t) d t \\
\leq & F\left(u\left(t_{2}\right)\right)+\int_{0}^{T} \frac{\alpha_{+}(t)}{u^{\mu}(t)} d t+\int_{0}^{T} \frac{\beta_{-}(t)}{u^{y}(t)} d t+\int_{0}^{T} \varphi_{-}(t) u^{m}(t) d t \\
\leq & F\left(u\left(t_{2}\right)\right)+\frac{T \overline{\alpha_{+}}}{u^{\mu}\left(t_{2}\right)}+\frac{T \overline{\beta_{-}}}{u^{y}\left(t_{2}\right)}+T \overline{\varphi_{-}} u^{m}\left(t_{3}\right),
\end{aligned}
$$


i.e.,

$$
F\left(u\left(t_{3}\right)\right)-T \overline{\varphi_{-}} u^{m}\left(t_{3}\right) \leq F\left(u\left(t_{2}\right)\right)+\frac{T \overline{\alpha_{+}}}{u^{\mu}\left(t_{2}\right)}+\frac{T \overline{\beta_{-}}}{u^{y\left(t_{2}\right)}},
$$

which together with (3.11) and gives

$$
F\left(u\left(t_{3}\right)\right)-T \overline{\varphi_{-}} u^{m}\left(t_{3}\right) \leq \sup _{x \in\left[y_{0}, A_{0}\right]}\left[F(x)+\frac{T \overline{\alpha_{+}}}{x^{\mu}}+\frac{T \overline{\beta_{-}}}{x^{y}}\right]:=C_{0}<+\infty .
$$

It follows from (3.10) and the condition $\bar{\varphi}>0$ that there is a constant $\rho_{2}>0$ such that

$$
\max _{t \in[0, T]} u(t)=u\left(t_{3}\right) \leq \rho_{2} .
$$

From (3.11) and (3.13), we see that the conclusions of (3.3) and (3.4) hold.

Theorem 3.3. Assume $\bar{\varphi}>0, \bar{\alpha}>0$ and

$$
\lim _{x \rightarrow+\infty}\left[G(x)-T \overline{\beta_{+}} x^{\mu-y}-T \overline{\varphi_{+}} x^{\mu+m}\right]=+\infty .
$$

If

$$
\lim _{x \rightarrow 0^{+}} G(x)<C_{0}-T \overline{\alpha_{-}},
$$

then equation(1.3) has at least one positive T-periodic solution, where $G(x)$ is defined by (2.4) and

$$
C_{0}:=\inf _{x \in\left[A_{1},+\infty\right)}\left[G(x)-T \overline{\beta_{+}} \chi^{\mu-y}-T \overline{\varphi_{+}} \chi^{\mu+m}\right] .
$$

Remark 3.1. Assumption (3.14) implies that $C_{0} \in(-\infty,+\infty)$ is a constant.

Proof. Similar to the proof of Theorem 3.2, we need only prove the estimates of (3.4) and (3.3). Let $u \in D$, then $u$ satisfies (2.18). Let $t_{1}$ and $t_{2}$ be as same as the ones in the proof of Lemma 2.4. Multiplying (2.18) with $u^{\mu}(t)$ and integrating it on $\left[t_{4}, t_{2}\right]$, we get

$$
\begin{aligned}
& \int_{t_{1}}^{t_{2}} u^{\prime \prime}(t) u^{\mu}(t) d t+\lambda \int_{t_{1}}^{t_{2}} u^{\mu}(t) f(u(t)) u^{\prime}(t) d t \\
& =\lambda \int_{t_{4}}^{t_{2}} \alpha(t) d t-\lambda \int_{t_{4}}^{t_{2}} \varphi(t) u^{m+\mu}(t) d t-\lambda \int_{t_{4}}^{t_{2}} \beta(t) u^{\mu-y}(t) d t .
\end{aligned}
$$

Since $\int_{t_{1}}^{t_{2}} u^{\prime \prime}(t) u^{\mu}(t) d t \leq 0$, it follows from (3.17) and (3.16) that

$$
\begin{aligned}
G\left(u\left(t_{2}\right)\right) & \geq G\left(u\left(t_{1}\right)\right)+\int_{t_{1}}^{t_{2}} \alpha(t) d t-\int_{t_{1}}^{t_{2}} \varphi_{+}(t) u^{m+\mu}(t) d t-\int_{t_{1}}^{t_{2}} \beta_{+}(t) u^{\mu-y}(t) d t \\
& \geq G\left(u\left(t_{1}\right)\right)-T \overline{\alpha_{-}}-T \overline{\varphi_{+}} u^{m+\mu}\left(t_{1}\right)-T \overline{\beta_{+}} u^{\mu-y}\left(t_{1}\right) .
\end{aligned}
$$

By using (2.6) and (3.16), we get

$$
\begin{aligned}
G\left(u\left(t_{2}\right)\right) \geq \inf _{x \in\left[A_{1},+\infty\right)}\left[G(x)-T \overline{\varphi_{+}} x^{m+\mu}-T \overline{\beta_{+}} x^{\mu-y}\right]-T \alpha_{-} \\
=C_{0}-T \overline{\alpha_{-}}
\end{aligned}
$$

which together with (3.15) verifies (3.4). Thus, according to (2.5), we arrive at

$$
y_{2} \leq u\left(t_{2}\right) \leq A_{0} .
$$


On the other hand, by (3.18) again, we obtain from (3.19) that

$$
\begin{aligned}
& G\left(u\left(t_{1}\right)\right)-T \overline{\varphi_{+}} u^{m+\mu}\left(t_{1}\right)-T \overline{\beta_{+}} u^{\mu-y}\left(t_{1}\right) \\
& \leq G\left(u\left(t_{2}\right)\right)+T \overline{\alpha_{-}} \\
& \leq \max _{y_{2} \leq x \leq A_{0}} G(x)+T \overline{\alpha_{-}} .
\end{aligned}
$$

By assumption (3.14), we see that there is a constant $y_{3}>y_{2}$ such that

$$
\begin{aligned}
& G(s)-T \overline{\varphi_{+}} s^{m+\mu}-T \overline{\beta_{+}} s^{\mu-y} \\
> & \max _{y_{2} \leq x \leq A_{0}} G(x)+T \overline{\alpha_{-}}, \text {for all } s \in\left(y_{3},+\infty\right) .
\end{aligned}
$$

Therefore, (3.20) and (3.21) imply

$$
\max _{t \in[0, T]} u(t)=u\left(t_{4}\right) \leq y_{3} .
$$

From (3.19) and (3.22), we see that the (3.4) and (3.3) hold.

Theorem 3.4. Assume $\bar{\varphi}>0, \bar{\alpha}>0$ and

$$
\lim _{x \rightarrow+\infty}\left[G(x)+T{\overline{\beta_{+}}}^{\mu-y}+T \overline{\varphi_{+}} x^{\mu+m}\right]=-\infty .
$$

If

$$
\lim _{x \rightarrow 0^{+}} G(x)>C_{1}+T \overline{\alpha_{-}},
$$

then equation(1.3) has at least one positive T-periodic solution, where

$$
C_{1}:=\sup _{x \in\left[A_{1},+\infty\right)}\left[G(x)+T \overline{\beta_{+}} x^{\mu-y}+T \overline{\varphi_{+}} x^{\mu+m}\right] .
$$

Remark 3.2. From assumption (3.23), one can find that $C_{1} \in(-\infty,+\infty)$ is a constant.

Proof. Let $u \in D$. If set $t_{4}=t_{2}-T$, then $0<t_{1}-t_{4} \leq T$ and $u\left(t_{4}\right)=\min _{t \in[0, T]} u(t)$. Multiplying (2.18) with $u^{\mu}(t)$ and integrating it on $\left[t_{4}, t_{1}\right]$, we get

$$
\begin{aligned}
& \int_{t_{4}}^{t_{1}} u^{\prime \prime}(t) u^{\mu}(t) d t+\lambda \int_{t_{4}}^{t_{1}} u^{\mu}(t) f(u(t)) u^{\prime}(t) d t \\
& =\lambda \int_{t_{4}}^{t_{1}} \alpha(t) d t-\lambda \int_{t_{4}}^{t_{1}} \varphi(t) u^{m+\mu}(t) d t-\lambda \int_{t_{4}}^{t_{1}} \beta(t) u^{\mu-y}(t) d t .
\end{aligned}
$$

By using $\int_{t_{4}}^{t_{1}} u^{\prime \prime}(t) u^{\mu}(t) d t \leq 0$, it follows from (3.26) that

$$
\begin{aligned}
G\left(u\left(t_{1}\right)\right) & \geq G\left(u\left(t_{4}\right)\right)+\int_{t_{4}}^{t_{1}} \alpha(t) d t-\int_{t_{4}}^{t_{1}} \varphi_{+}(t) u^{m+\mu}(t) d t-\int_{t_{4}}^{t_{1}} \beta_{+}(t) u^{\mu-y}(t) d t \\
& \geq G\left(u\left(t_{4}\right)\right)-T \overline{\alpha_{-}}-T \overline{\varphi_{+}} u^{m+\mu}\left(t_{1}\right)-T \overline{\beta_{+}} u^{\mu-y}\left(t_{1}\right),
\end{aligned}
$$

which together with (3.25) gives

$$
\begin{aligned}
& G\left(u\left(t_{4}\right)\right) \\
\leq & G\left(u\left(t_{1}\right)\right)+T \overline{\varphi_{+}} u^{m+\mu}\left(t_{1}\right)+T \overline{\beta_{+}} u^{\mu-y}\left(t_{1}\right)+T \overline{\alpha_{-}} \\
\leq & \sup _{x \in\left[A_{1},+\infty\right)}\left[G(x)+T \overline{\beta_{+}} x^{\mu-y}+T \overline{\varphi_{+}} x^{\mu+m}\right]+T \overline{\alpha_{-}} \\
\leq & C_{1}+T \overline{\alpha_{-}} .
\end{aligned}
$$


In view of (3.24), we get that there is a constant $y_{4}>0$ such that

$$
\min _{t \in[0, T]} u(t)>y_{4}
$$

This together with (2.5) yields

$$
y_{4} \leq u\left(t_{4}\right) \leq A_{0} .
$$

It follows from (3.27) that

$$
\begin{aligned}
& G\left(u\left(t_{1}\right)\right)+T \overline{\varphi_{+}} u^{m+\mu}\left(t_{1}\right)+T \overline{\beta_{+}} u^{\mu-y}\left(t_{1}\right)+T \alpha_{-} \\
\geq & G\left(u\left(t_{4}\right)\right)-T \overline{\alpha_{-}} \\
\geq & \min _{y_{4} \leq x \leq A_{0}} G(x)-T \overline{\alpha_{-}},
\end{aligned}
$$

to which by applying (3.23), we get that there is a constant $y_{5}>y_{4}$ such that

$$
\max _{t \in[0, T]} u(t)=u\left(t_{1}\right)<y_{5}
$$

Example 3.1: Consider the following equation

$$
x^{\prime \prime}(t)-\left(x^{m}+\frac{1}{x^{2}}\right) x^{\prime}(t)+(1+2 \sin t) x^{m}(t)-\frac{1-2 \cos t}{x^{\frac{2}{3}}(t)}+\frac{\sin t}{x^{\frac{1}{2}}(t)}=0,
$$

where $m \in[0,+\infty)$ is a constant.

Corresponding to (1.3), we have $f(x)=-x^{m}-\frac{1}{x^{2}}, \mu=\frac{2}{3}, y=\frac{1}{2}, \varphi(t)=1+2 \sin t, \alpha(t)=1-2 \cos t$, $\beta(t)=\sin t$ and $T=2 \pi$. Clearly, $\bar{\alpha}=1>0$ and $\bar{\varphi}=1>0$. Since $A_{1}=\left(\frac{\bar{\alpha}}{\bar{\varphi}}\right)^{\frac{1}{m+\mu}}=1$ and

$$
F(x)=\int_{1}^{x} f(s) d s=-\frac{x^{m+1}}{m+1}+\frac{1}{x}-\frac{m}{m+1},
$$

we have

$$
\lim _{x \rightarrow+\infty}\left(F(x)+T \overline{\varphi_{+}} x^{m}\right)=-\infty .
$$

Furthermore, from $\overline{\alpha_{+}}=\frac{5 \pi}{3}-2$ and $\mu=\frac{2}{3}<1$, we have

$$
\lim _{s \rightarrow 0^{+}}\left(F(s)-\frac{T \overline{\alpha_{+}}}{s^{\mu}}-\frac{T \overline{\beta_{-}}}{s^{y}}\right)=+\infty,
$$

Thus, by using Theorem 3.1, we have that equation(3.28) has at least one positive $2 \pi$-periodic solution.

Now, we study the existence of periodic solutions to equation (1.4). By using the change of variable $R=$ $x^{\frac{2}{5}}$, from [5], we see that equation (1.4) changes to

$$
x^{\prime \prime}+\frac{4 v}{x^{\frac{4}{5}}} x^{\prime}+\frac{5\left[P_{\infty}(t)-P_{v}\right]}{2 \rho} x^{\frac{1}{5}}+\frac{5 S}{x^{\frac{1}{5}}}-\left(\frac{5 P_{g_{0}} R_{0}^{3 k}}{2 \rho}\right) \frac{1}{x^{\frac{6 k-1}{5}}}=0,
$$

where the parameters of $S, \rho, v, P_{g_{0}}$ and $R_{0}$ are positive constants, $k \in\left(\frac{1}{3},+\infty\right)$. Corresponding to (1.3), we have $f(x)=\frac{4 v}{x^{4}}, \varphi(t)=\frac{5\left[P_{\infty}(t)-P_{v}\right]}{2 \rho}, \alpha(t) \equiv \frac{5 P_{g_{0}} R_{0}^{3 k}}{2 \rho}, \beta(t) \equiv 5 S, \mu=\frac{6 k-1}{5}$ and $y=m=\frac{1}{5}$. Thus,

$$
G(x)=\int_{1}^{x} s^{\mu} f(s) d s=4 v \int_{1}^{x} s^{\frac{6 k-1}{5}-\frac{4}{5}} d s=\frac{10 v}{3 k} x^{\frac{6 k}{5}}-\frac{10 v}{3 k},
$$


and from (2.7), we see

$$
\begin{aligned}
A_{1} & =\min \left\{\left(\frac{\bar{\alpha}}{2 \overline{\varphi_{+}}}\right)^{\frac{1}{\mu+m}},\left(\frac{\bar{\alpha}}{2 \overline{\beta_{+}}}\right)^{\frac{1}{\mu-y}}\right\} \\
& =\min \left\{\left(\frac{P_{g_{0}} R_{0}^{3 k}}{2 \overline{\left[P_{\infty}(t)-P_{V}\right]_{+}}}\right)^{\frac{1}{\mu+m}},\left(\frac{P_{g_{0}} R_{0}^{3 k}}{2 S}\right)^{\frac{1}{\mu-y}}\right\} .
\end{aligned}
$$

If $\frac{10 v}{3 k}>\frac{5 T}{2 \rho} \overline{\left[P_{\infty}(t)-P_{v}\right]_{+}}$, then

$$
G(x)-T \overline{\beta_{+}} \chi^{\mu-y}-T \overline{\varphi_{+}} \chi^{\mu+m}=\left(\frac{10 v}{3 k}-\frac{5 T}{2 \rho} \overline{\left[P_{\infty}(t)-P_{v}\right]_{+}}\right) x^{\frac{6 k}{5}}-5 T S x^{\frac{6 k-2}{5}}-\frac{10 v}{3 k},
$$

and so assumption (3.14) holds. Let

$$
H(x)=\left(\frac{10 v}{3 k}-\frac{5 T}{2 \rho} \overline{\left[P_{\infty}(t)-P_{v}\right]_{+}}\right) x^{\frac{6 k}{5}}-5 T S x^{\frac{6 k-2}{5}} .
$$

Clearly, if $A_{1}>\left(\frac{5 T S}{\sigma_{1}}\right)^{\frac{5}{2}}$, then

$$
\begin{aligned}
C_{0} & :=\inf _{x \in\left[A_{1},+\infty\right)}\left[G(x)-2 T \overline{\beta_{+}} x^{\mu-y}-2 T \overline{\varphi_{+}} x^{\mu+m}\right] \\
& =\inf _{x \in\left[A_{1},+\infty\right)} H(x)-\frac{10 v}{3 k} \\
& =H\left(A_{1}\right)-\frac{10 v}{3 k} \\
& >-\frac{10 v}{3 k},
\end{aligned}
$$

where

$$
\sigma_{1}=\frac{10 v}{3 k}-\frac{5 T}{2 \rho} \overline{\left[P_{\infty}(t)-P_{v}\right]_{+}}
$$

Furthermore,

$$
\lim _{x \rightarrow 0^{+}} G(x)=-\frac{10 v}{3 k},
$$

which together with (3.33) yields

$$
\lim _{x \rightarrow 0^{+}} G(x)<C_{0}-T \alpha_{-} .
$$

This implies that assumption (3.15) holds. Thus, By using Theorem 3.3, we obtain the following result.

Theorem 3.5. Assume $k \in\left(\frac{1}{3},+\infty\right), \overline{P_{\infty}}>P_{v}$. If $\frac{10 v}{3 k}>\frac{5 T}{2 \rho} \overline{\left[P_{\infty}(t)-P_{v}\right]_{+}}$, and

$$
A_{1}>\left(\frac{5 T S}{\sigma_{1}}\right)^{\frac{5}{2}}
$$

then (3.31) has at least one positive T-periodic solution, where $\sigma_{1}$ and $A_{1}$ is defined by (3.34) and (3.32), respectively.

Remark 3.2: Since the condition $\overline{P_{\infty}}>P_{V}$ in Theorem 3.5 is essentially different from the corresponding one of $\overline{P_{\infty}}<P_{v}$ in Theorem 1.1, Theorem 1.4 can be regarded as a new result on the existence of periodic solutions to Rayleigh-Plesset equation (1.4).

Acknowledgement: This work was sponsored by NSF of China (No. 11271197).

\section{References}

[1] J. Chu, P. J. Torres and M. Zhang, Periodic solutions of second order non-autonomous singular dynamical systems, J. Differential Equations 239 (2007), no.1, 196-212. 
[2] A. Fonda and A.Sfecci, On a singular periodic Ambrosetti-Prodi problem, Nonlinear Anal. 149 (2017), 146-155.

[3] P. Habets and L. Sanchez, Periodic solutions of some Liénard equations with singularities, Proc. Amer. Math. Soc. 109 (1990), no.4, 1135-1144.

[4] R. Hakl, P. J. Torres, On periodic solutions of second order differential equations with attractive-repulsive singularities, J. Differential Equations 248 (2010), no.1111-126.

[5] R. Hakl, P.J. Torres and M. Zamora, Periodic solutions of singular second order differential equations: the repulsive case, Topol. Methods Nonlinear Anal. 39( 2012), no.2, 199-220.

[6] P. Jebelean and J. Mawhin, Periodic solutions of singular nonlinear perturbations of the ordinary p-Laplacian, Adv. Nonlinear Stud. 2 (2002), no.3, 299-312.

[7] R. Martins, Existence of periodic solutions for second-order differential equations with singularities and the strong force condition, J. Math. Anal. Appl. 317 (2006), no.1, 1-13.

[8] P.J. Torres, Existence of one-signed periodic solutions of some second order differential equations via a Krasnoselskii fixed point theorem, J. Differential Equations, 190 (2003), no.2, 643-662.

[9] P.J. Torres, Bounded solutions in singular equations of repulsive type, Nonlinear Anal. 32 (1998), no.1, 117-125.

[10] P.J. Torres, Weak singularities may help periodic solutions to exist, J. Differential Equations 232 (2007), no.1, 277-284.

[11] P.J. Torres and M. Zhang, Twist periodic solutions of repulsive singular equations, Nonlinear Anal. 56 (2004), no.4, 591 599.

[12] A. J. Ureña, Periodic solutions of singular equations, Topol. Methods Nonlinnear Anal. 47 (2016), no.1, 55-72.

[13] Z. Wang, Periodic solutions of Liénard equation with a singularity and a deviating argument, Nonlinear Anal. Real World Appl. 16 (2014), 227-234.

[14] M. Zhang, Periodic solutions of Liénard equations with singular forces of repulsive type, J. Math. Anal. Appl. 203 (1996), no.1, 254-269.

[15] R. Hakl and P. J. Torres, On periodic solutions of second order differential equations with attractive-repulsive singularities, J. Differential Equations, 248 (2010), no.1, 111-126.

[16] J. Chu, P.J. Torres and F. Wang, Twist periodic solutions for differential equations with a combined attractive Crepulsive singularity, J. Math. Anal. Appl. 437 (2016), no.2, 1070-1083.

[17] J. L. Bravo and P. J. Torres, Periodic solutions of a singular equation with indefinite weight, Adv. Nonlinear Stud. 10 (2010), no.4, 927-938.

[18] R. Hakl and M. Zamora, Periodic solutions to second-order indefinite singular equations, J. Differential Equations 263 (2017), no.1, 451-469.

[19] R. Hakl and M. Zamora, Existence and Multiplicity of Periodic solutions to indefinite singular equations having a nonmonotone term with two singularities, Adv. Nonlinear Stud. 19 (2018), no.2, 317-322.

[20] S. Lu, Y. Guo and L. Chen, Periodic solutions for Liénard equation with an indefinite singularity, Nonlinear Anal. Real World Appl. 45 (2019), 542-556.

[21] 20 P.J. Torres, Mathematical Models with Singularities -A Zoo of Singular Creatures. Atlantis Press, Amsterdam (2015). ISBN:978-94-6239-105-5.

[22] M. P. Brenner, S. Hilgenfeldt and D. Lohse, Single bubble sonoluminescence, Rev. Modern Phys. 74 (2002), no.2, 425-484.

[23] J. P. Franc, The Rayleigh-Plesset equation: A simple and powerful tool to understand various aspects of cavitation. In Fluid Dynamics of Cavitation and Cavitating Turbopumps, Springer Vienna, 2008.

[24] R. Hakl, P. J. Torres and M. Zamora, Periodic solutions of singular second order differential equations: Upper and lower functions, Nonlinear Anal. 74 (2011), no.18, 7078-7093.

[25] M. S. Plesset, The dynamics of cavitation bubbles, ASME J. Appl. Mech., 16 (1949) 228-231.

[26] R. Manásevich and J. Mawhin, Periodic solutions for nonlinear systems with p-Laplacian-like operators, J. Differential Equations, 145 (1998), no.2, 367-393. 